\title{
Validação de marcadores moleculares para resistência à giberela em genótipos brasileiros de trigo
}

\author{
Adriana Scherloski( ${ }^{(1)}$, Tatiane Dalla Nora Montecelli(2), Franciele Mioranza( ${ }^{(2)}$, Fabiane Lazzari ${ }^{(2)}$, \\ Leandra Regina Texeira Polo(2) e Ivan Schuster ${ }^{(2)}$
}

\begin{abstract}
(1)Universidade Paranaense, Praça Mascarenhas de Moraes, № 4.282, Zona III, CEP 87502-210, Umuarama, PR, Brasil. E-mail: scherloski.a@gmail.com (2)Cooperativa Central de Pesquisa Agrícola, Rodovia BR 467, Km 98, Zona Rural, CEP $85813-450$ Cascavel, PR, Brasil. E-mail: tatianedn@coodetec.com.br, fmioranza@coodetec.com.br, flazzari@coodetec.com.br, leandra.texeira@coodetec.com.br, ivan@coodetec.com.br
\end{abstract}

Resumo - O objetivo deste trabalho foi validar 19 marcadores microssatélites para resistência do trigo à giberela, em uma população não estruturada. Foram utilizados marcadores moleculares descritos na literatura como flanqueando QTLs de resistência à giberela em trigo, nos cromossomos 3B, 5A e 6B. Foram avaliadas 96 linhagens e cultivares de trigo quanto à severidade da infecção por giberela, em dois anos de avaliação. As linhagens e as cultivares foram genotipadas com 19 marcadores microssatélites. Os dados obtidos foram analisados pelo teste de Tukey e pelas análises de correlação, regressão linear simples e regressão múltipla; também foi estimada a eficiência de seleção dos marcadores moleculares. A severidade da doença variou de 1,95 a 41,3\%, na média dos dois anos. Foram validados os QTLs nos três cromossomos avaliados. Os marcadores Xgwm389, Xgwm533, Xbarc180, Xbarc24, Wmc397, Xbarc101 e Wmc398 foram associados significativamente à resistência do trigo à giberela, tendo sido identificados alelos de resistência $\mathrm{e}$ de suscetibilidade. Os marcadores Wmc397, Xbarc101 (cromossomo 6B) e Xbarc180 (cromossomo 5A) têm potencial para uso na seleção assistida por marcadores moleculares, para resistência do trigo à giberela.

Termos para indexação: Fusarium graminearum, Triticum aestivum, seleção assistida por marcadores moleculares, SAM.

\section{Validation of molecular markers for wheat resistance to Fusarium head blight in Brazilian wheat genotypes}

\begin{abstract}
The objective of this work was to validate 19 microsatellite markers for wheat resistance to Fusarium head blight (FHB), in an unstructured population. Molecular markers described in the literature as flanking QTLs of FHB resistance in wheat on chromosomes 3B, 5A, and 6B were used. Ninety six wheat lines and cultivars were evaluated for severity of FHB infection in two evaluation years. The lines and cultivars were genotyped with 19 microsatellite markers. The obtained data were analyzed by Tukey's test, and by correlation, simple linear regression, and multiple regression analyses; the efficiency of selection with molecular markers was also estimated. Disease severity ranged from 1.95 to $41.3 \%$ in the mean of the two years. QTLs in the three evaluated chromosomes were validated. The markers Xgwm389, Xgwm533, Xbarc180, Xbarc24, Wmc397, Xbarc101, and Wmc398 were significantly associated with FHB resistance in wheat, and alleles for susceptibility and resistance were identified. The markers Wmc397, Xbarc101 (chromosome 6B), and Xbarc180 (chromosome 5A) have potential for use in marker-assisted selection for resistance to FHB in wheat.

Index terms: Fusarium graminearum, Triticum aestivum, marker-assisted selection, MAS.
\end{abstract}

\section{Introdução}

A giberela ou a fusariose é uma das principais doenças do trigo (Triticum aestivum L.). É causada, principalmente, pelo fungo Fusarium graminearum Schwabe e ocorre em regiões onde o clima é quente e úmido (Casa et al., 2004). Além de reduzir a produtividade do trigo, o fungo produz a micotoxina deoxinivalenol (DON), que é prejudicial tanto para a saúde humana quanto a animal (Draeger et al., 2007).

Até o momento, vários QTLs relacionados à resistência do trigo à giberela foram identificados. O QTL que apresenta maior efeito foi mapeado no braço curto do cromossomo 3B da variedade chinesa 
Sumais 3 e passou a ser denominado de $F h b 1$ ou $Q f h s$. ndsu-3BS (Cuthbert et al., 2006; Anderson et al., 2007; Pumphrey et al., 2007).

De acordo com Liu \& Anderson (2003) e Cuthbert et al. (2006), o QTL no loco Fhbl encontra-se flanqueado pelos marcadores Xgwm493 e Xgwm533. Anderson et al. (2001), ao avaliar esta região em uma população derivada de Sumai 3/Stoa, constataram que o QTL explica até $42 \%$ da variação fenotípica para resistência à giberela. Essa região do cromossomo 3B está relacionada à resistência do tipo II, ou seja, à resistência à disseminação do fungo na espiga (Anderson et al., 2001; Zhou et al., 2004).

Cuthbert et al. (2006), ao utilizar Sumai 3 como fonte de resistência para giberela, mapearam outro QTL relacionado à resistência do tipo II - Fhb2 -, localizado no braço curto do cromossomo $6 \mathrm{~B}$ e flanqueado pelos marcadores Xgwm133 e Xgwm644. Xue et al. (2011) mapearam o QTL Fhb5, proveniente de Wangshuibai. Esse QTL encontra-se flanqueado pelos marcadores Xgwm304 e Xgwm415 no cromossomo 5A e está relacionado à resistência do tipo $\mathrm{I}$, à infecção inicial.

Para serem utilizados na seleção assistida por marcadores moleculares em programas de melhoramento, os marcadores publicados na literatura devem ser primeiramente validados em populações representativas do material de melhoramento rotineiramente selecionado (Schuster, 2011).

O objetivo deste trabalho foi validar 19 marcadores microssatélites para resistência do trigo à giberela, em uma população não estruturada.

\section{Material e Métodos}

Foram utilizadas 13 cultivares comerciais de trigo brasileiras (CD 1252, CD 150, CD 1550, BRS 177, BRS 179, BRS Camboim, BRS Guamirim, BRS Pardela, FUNDACEP Raízes, FUNDACEP 52, Ônix, Quartzo e Esporão), uma cultivar de domínio público (Frontana), três cultivares exóticas (Kasoro, Ning7840 e Weebill) e 76 linhagens experimentais de trigo, o que totalizou 96 acessos. As sementes foram obtidas no Banco de Germoplasma de Trigo da Cooperativa Central de Pesquisa Agrícola (Coodetec). As cultivares e as linhagens foram avaliadas quanto ao seu nível de resistência à giberela, nas safras de 2010 e 2011, em condições controladas, no Centro de Pesquisas da Coodetec, em Cascavel, PR (245'ㄷㄴ"S, $53^{\circ} 32^{\prime} 0^{\prime \prime} \mathrm{W}$, a $690 \mathrm{~m}$ de altitude).
Cada variedade ou linhagem foi semeada manualmente em uma linha de $1 \mathrm{~m}$ de comprimento, compondo parcelas de três linhas, sendo que, em cada linha lateral, foi semeada uma variedade ou uma linhagem; na linha central de cada parcela, foi semeada a testemunha suscetível 'CD 116', para monitoramento da inoculação e do desenvolvimento da doença no campo experimental. O espaçamento entre as linhas foi de $20 \mathrm{~cm}$ e entre as parcelas, de $40 \mathrm{~cm}$. A adubação e o controle de pragas foram efetuados de acordo com as exigências técnicas para a cultura, sendo realizada apenas uma aplicação do fungicida Tebuconazole na dose de $0,5 \mathrm{~L} \mathrm{ha}^{-1}$ do produto comercial, no estádio de alongamento, para controle das doenças foliares.

A produção de inóculo foi iniciada com a multiplicação do isolado de $F$. graminearum, em meio de cultura BDA, em câmara de crescimento sob luz contínua e $25 \pm 1^{\circ} \mathrm{C}$, durante sete dias.

Foram preparados $300 \mathrm{~g}$ de trigo em cada frasco Erlenmeyer de $500 \mathrm{~mL}$ de capacidade. Os grãos permaneceram em repouso durante uma noite, embebidos em água; em seguida, foi escoado o excesso de água dos grãos, os quais foram esterilizados em autoclave por duas vezes a $125^{\circ} \mathrm{C}$, durante $20 \mathrm{~min}$, em dois dias.

Após o resfriamento, os grãos de trigo foram inoculados pela adição de $10 \mathrm{~mL}$ de suspensão contendo aproximadamente $1,0 \times 10^{6}$ macroconídios por $\mathrm{mL}$ do isolado de $F$. graminearum, anteriormente multiplicado em meio de cultura BDA.

Os frascos foram incubados em câmara de crescimento durante aproximadamente três semanas, a $25 \pm 2^{\circ} \mathrm{C}$, com fotoperíodo de 12 horas, para colonização dos grãos.

Os grãos de trigo colonizados com o micélio do fungo foram colocados em bandejas de plástico contendo areia esterilizada e foram molhados diariamente, para que a umidade fosse mantida constante.

As bandejas com o inóculo ficaram expostas ao sol ou à luz artificial para favorecer a formação de peritécios de Gibberella zeae. No início da formação de peritécios nos grãos, aproximadamente aos 21 dias, os grãos de trigo colonizados com os peritécios foram distribuídos entre as linhas externas de cada parcela, quando as primeiras plantas entraram no estádio de espigamento. No início do florescimento do trigo, a área experimental foi submetida ao molhamento das espigas com formação de neblina, por $5 \mathrm{~min}$ 
consecutivos, a intervalos de 25 a $30 \mathrm{~min}$, em dias sem precipitação pluvial.

A severidade foi avaliada em 20 espigas principais colhidas no estádio 11.4 da escala modificada de Feekes-Large (Large, 1954), isto é, de espiga verde, e o percentual da severidade foi determinado conforme escala proposta por Stack \& McMullen (1995). De acordo com a severidade observada nas cultivares conhecidas, consideraram-se resistentes as cultivares e as linhagens com severidade igual ou menor do que $5 \%$, e suscetíveis aquelas com severidade maior do que $5 \%$. Para as análises quantitativas, no cálculo da percentagem de infecção, foram utilizadas as médias dos dois anos avaliados. Para a avaliação qualitativa, as linhagens que apresentaram menos de 5\% de infecção em um ano de avaliação e mais de $5 \%$ no outro ano foram classificadas como intermediárias.

Aextração de DNA foi realizada conforme descrito por Schuster et al. (2009). Foram utilizados 19 marcadores microssatélites, localizados nos cromossomos 3B, $5 \mathrm{~A}$ e 6B do trigo, relatados na literatura como ligados a QTLs de resistência à giberela (Tabela 1).

Tabela 1. Descrição dos marcadores moleculares utilizados na validação para resistência do trigo (Triticum aestivum) à giberela, e origem da informação de associação do marcador com a resistência.

\begin{tabular}{|c|c|c|c|c|}
\hline Marcador & Cromossomo & Iniciador para $\mathrm{PCR}^{(1)}$ & Referência & QTL $(\%)^{(2)}$ \\
\hline Xgwm533 & $3 \mathrm{~B}$ & $\begin{array}{l}\text { 5'AAGGCGAATCAAACGGAATA 3' } \\
\text { 5' GTTGCTTTAGGGGAAAAGCC 3' }\end{array}$ & $\begin{array}{l}\text { Anderson et al. (2001) } \\
\text { Burlakoti et al. (2010) }\end{array}$ & $\begin{array}{l}24,8-41,6 \% \\
\text { SI }\end{array}$ \\
\hline Xgwm389 & $3 \mathrm{~B}$ & $\begin{array}{l}\text { 5' ATCATGTCGATCTCCTTGACG 3' } \\
\text { 5' TGCCATGCACATTAGCAGAT 3' }\end{array}$ & $\begin{array}{l}\text { Shi et al. (2008) } \\
\text { Xue et al. (2010) }\end{array}$ & $\begin{array}{l}\text { SI } \\
\text { SI }\end{array}$ \\
\hline Xbarc68 & $3 \mathrm{~B}$ & $\begin{array}{c}\text { 5' CGATGCCAACACACTGAGGT 3', } \\
\text { 5'AGCCGCATGAAGAGATAGGTAGAGAT 3, }\end{array}$ & $\begin{array}{l}\text { Shi et al. (2008) } \\
\text { Cuthbert et al. (2006) }\end{array}$ & $\begin{array}{l}\text { SI } \\
\text { SI }\end{array}$ \\
\hline Xbarc133 & $3 \mathrm{~B}$ & $\begin{array}{l}\text { 5' AGCGCTCGAAAAGTCAG 3' } \\
\text { 5' GGCAGGTCCAACTCCAG 3' }\end{array}$ & $\begin{array}{l}\text { Shen et al. (2003) } \\
\text { Burlakoti et al. (2010) }\end{array}$ & $\begin{array}{l}19,2-40 \% \\
\text { SI }\end{array}$ \\
\hline Xgwm493 & $3 \mathrm{~B}$ & $\begin{array}{l}\text { 5', TTCCCATAACTAAAACCGCG 3', } \\
\text { 5' GGAACATCATTTCTGGACTTTG 3' }\end{array}$ & $\begin{array}{l}\text { Anderson et al. (2001) } \\
\text { Burlakoti et al. (2010) }\end{array}$ & $\begin{array}{l}24.8-41,6 \% \\
\text { SI }\end{array}$ \\
\hline Xbarc147 & $3 \mathrm{~B}$ & $\begin{array}{l}\text { 5' GCGCCATTTATTCATGTTCCTCAT 3', } \\
\text { 5' CCGCTTCACATGCAATCCGTTGAT 3' }\end{array}$ & Burlakoti et al. (2010) & $\begin{array}{l}18-48 \% \\
\text { SI }\end{array}$ \\
\hline Xbarc75 & $3 \mathrm{~B}$ & $\begin{array}{l}\text { 5' AGGGTTACAGTTTGCTCTTTTAC 3', } \\
\text { 5' CCCGACGACCTATCTATACTTCTCTA 3, }\end{array}$ & $\begin{array}{c}\text { Zhou et al. (2003) } \\
\text { Liu \& Anderson (2003) }\end{array}$ & $\begin{array}{l}\text { SI } \\
\text { SI }\end{array}$ \\
\hline Xgwm293 & $5 \mathrm{~A}$ & $\begin{array}{l}\text { 5' TACTGGTTCACATTGGTGCG 3' } \\
\text { 5' TCGCCATCACTCGTTCAAG 3' }\end{array}$ & $\begin{array}{l}\text { Buerstmayr et al. (2002) } \\
\text { Cuthbert et al. (2006) }\end{array}$ & $\begin{array}{l}\text { SI } \\
\text { SI }\end{array}$ \\
\hline Xbarc180 & $5 \mathrm{~A}$ & $\begin{array}{l}\text { 5' GCGATGCTTGTTTGTTACTTCTC 3', } \\
\text { 5' GCGATGGAACTTCTTTTTGCTCTA 3' }\end{array}$ & $\begin{array}{c}\text { Buerstmayr et al. (2002) } \\
\text { Shi et al. (2008) } \\
\text { Zhou et al. (2002) }\end{array}$ & $\begin{array}{l}\text { SI } \\
\text { SI } \\
\text { SI }\end{array}$ \\
\hline Xgwm639 & $5 \mathrm{~A}$ & $\begin{array}{c}\text { 5' CTCTCTCCATTCGGTTTTCC 3' } \\
\text { 5' CATGCCCCCCTTTTCTG 3' }\end{array}$ & Gervais et al. (2003) & $10,1-18,6 \%$ \\
\hline Xbarc197 & $5 \mathrm{~A}$ & $\begin{array}{l}\text { 5' CGCATGGTCAGTTTTCTTTTAATCCT 3' } \\
\text { 5' GCGCTCTCCTTCATTTATGGTTTGTTG 3', }\end{array}$ & Burlakoti et al. (2010) & SI \\
\hline Xbarc56 & $5 \mathrm{~A}$ & $\begin{array}{l}\text { 5' GCGGGAATTTACGGGAAGTCAAGAA 3' } \\
\text { 5' GCGAGTGGTTCAAATTTATGTCTGT 3' }\end{array}$ & Liu et al. (2009) & SI \\
\hline Xbarc101 & $6 \mathrm{~B}$ & $\begin{array}{c}\text { 5' GCTCCTCTCACGATCACGCAAAG 3', } \\
\text { 5' GCGAGTCGATCACACTATGAGCCAATG 3' }\end{array}$ & Anderson et al. (2001) & $4,9-9,2 \%$ \\
\hline Xgwm88 & $6 \mathrm{~B}$ & $\begin{array}{l}\text { 5' CACTACAACTATGCGCTCGC 3', } \\
\text { 5' TCCATTGGCTTCTCTCTCAA 3' }\end{array}$ & Shen et al. (2003) & $2,9-3,4 \%$ \\
\hline Wmc105 & $6 \mathrm{~B}$ & $\begin{array}{l}\text { 5' AATGTCATGCGTGTAGTAGCCA 3' } \\
\text { 5'AAGCGCACTTAACAGAAGAGGG 3', }\end{array}$ & Gosman et al. (2007) & SI \\
\hline Wmc397 & $6 \mathrm{~B}$ & $\begin{array}{l}\text { 5' AGTCGTGCACCTCCATTTTG 3', } \\
\text { 5' CATTGGACATCGGAGACCTG 3' }\end{array}$ & $\begin{array}{l}\text { Somers et al. (2006) } \\
\text { Burlakoti et al. (2010) }\end{array}$ & $23 \%$ \\
\hline Wmc539 & $6 \mathrm{~B}$ & $\begin{array}{l}\text { 5' GCAAGTAGGACCTTACAGTTCT 3' } \\
\text { 5' GTTATAACCTTTGTCCCTTCAC 3' }\end{array}$ & Lin et al. (2004) & SI \\
\hline Xbarc24 & $6 \mathrm{~B}$ & $\begin{array}{c}\text { 5' CGCCTCTTATGGACCAGCCTAT 3', } \\
\text { 5' GCGGTGAGCCATCGGGTTACAAAG 3, }\end{array}$ & Cuthbert et al. (2006) & SI \\
\hline Wmc398 & $6 \mathrm{~B}$ & $\begin{array}{l}\text { 5' GGAGATTGACCGAGTGGAT 3' } \\
\text { 5' CGTGAGAGCGGTTCTTTG 3' }\end{array}$ & Burlakoti et al. (2010) & SI \\
\hline
\end{tabular}

${ }^{(1)}$ De acordo com a base de dados GrainGenes do Departamento de Agricultura dos Estados Unidos (United States Departament of Agriculture, 2014). (2)Percentagem da variação na resistência à giberela, explicada pelo QTL, segundo a literatura citada. SI, sem informação. 
A amplificação foi realizada em $20 \mu \mathrm{L}$ de uma solução contendo $12,5 \mathrm{mmol} \mathrm{L}^{-1}$ de Tris- $\mathrm{HCl}(\mathrm{pH} 8,3)$, $62,5 \mathrm{mmol} \mathrm{L}^{-1}$ de $\mathrm{KCl}, 2,5 \mathrm{mmol} \mathrm{L}{ }^{-1}$ de $\mathrm{MgCl}_{2}$, $125 \mu \mathrm{mol} \mathrm{L}^{-1}$ de cada deoxinucleotídeo (dATP, dCTP, dTTP e dGTP), $0,4 \mu \mathrm{mol} \mathrm{L}^{-1}$ de cada iniciador, uma unidade da enzima Taq DNA polimerase e $75 \mathrm{ng}$ de DNA. O programa de ciclagem consistiu de uma etapa inicial de 7 min a $72^{\circ} \mathrm{C}$, seguida de 30 ciclos de $1 \mathrm{~min}$ a $94^{\circ} \mathrm{C}, 1 \mathrm{~min}$ a $55^{\circ} \mathrm{C}$ e $2 \min$ a $72^{\circ} \mathrm{C}$, e de uma etapa final de 7 min a $72^{\circ} \mathrm{C}$.

A separação dos fragmentos amplificados foi realizada em gel desnaturante de acrilamida $6 \%$. A corrida do gel foi realizada com carga elétrica de 300 volts por 2 horas e 30 min, e, em seguida, os géis foram corados com solução de nitrato de prata. Após corados, os géis foram digitalizados.

Para a codificação dos resultados, denominou-se de alelo 1 aquele que se localizou na porção superior do gel, e os demais foram denominados de alelo 2, 3 ou 4, de acordo com a posição, abaixo do alelo 1 (Figura 1). Para a avaliação dos haplótipos, cada alelo foi considerado como um marcador molecular, sendo que a presença do alelo em homozigose foi codificada com 2, e a ausência, com 0. Algumas linhagens ou variedades apresentaram mais de um alelo em determinados locos. Esses dados foram ignorados na

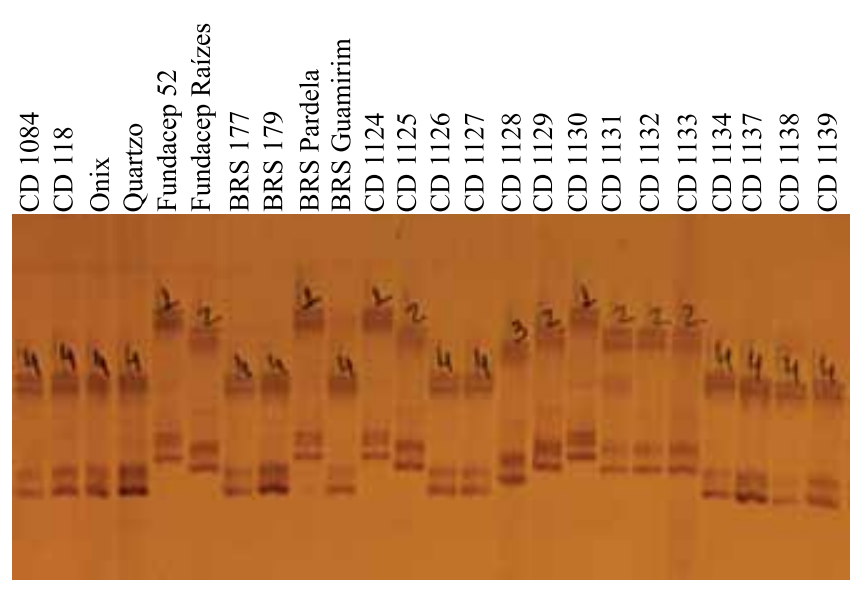

Figura 1. Identificação dos alelos do marcador Xgwm389, em parte da população estudada, no gel desnaturante de acrilamida $6 \%$. Alelos numerados de acordo com a posição no gel, em que o alelo 1 é o de maior tamanho, localizado mais acima no gel, seguido dos alelos 2,3 e 4, conforme a posição abaixo do alelo 1 . $\mathrm{O}$ alelo 1 está associado à resistência. análise dos dados, para que cada alelo fosse avaliado individualmente, sem o efeito de outros alelos.

Os resultados das médias da severidade de infecção por giberela, nos dois anos avaliados, foram submetidos à análise de variância e ao teste de Tukey, a 5\% de probabilidade, com uso do programa Genes (Cruz, 2006). Utilizou-se o delineamento inteiramente casualizado, em que os tratamentos foram constituídos pelos genótipos dos marcadores. Assim, para cada marcador, o grupo de indivíduos que apresentava o mesmo alelo foi considerado o mesmo tratamento, e a variável foi a severidade média da giberela nos dois anos de avaliação.

Os resultados também foram submetidos a análises de correlação e de regressão linear simples, para todos os marcadores, e de regressão múltipla, para os marcadores significativos na análise de correlação, tendo-se utilizado o programa JMP (SAS Institute, Cary, NC, EUA). Nas análises de correlação e de regressão dos marcadores com a reação de resistência e suscetibilidade das linhagens e das variedades de trigo, as plantas foram codificadas como: 2 , suscetíveis; 1 , intermediárias; e 0 , resistentes. Com essa codificação, alelos associados à resistência apresentam sinal negativo no coeficiente angular da regressão, e alelos associados à suscetibilidade apresentam sinal positivo, tanto na avaliação da severidade quanto na avaliação da reação.

$\mathrm{O}$ valor de probabilidade $(\mathrm{P})$ da correlação foi obtido a partir da distribuição de qui-quadrado. $\mathrm{O}$ valor da correlação ao quadrado $\left(\mathrm{r}^{2}\right)$ ponderado por $\mathrm{n}-1$ tem distribuição de qui-quadrado: $\chi^{2}=(n-1) r^{2}$, com um grau de liberdade. Os alelos com frequência menor do que $5 \%$ não foram utilizados nas análises.

A eficiência de seleção (ES) também foi estimada para a seleção realizada exclusivamente pelos marcadores moleculares, por meio da expressão:

$\mathrm{ES}=$ número de acertos do fenótipo com uso de marcadores moleculares/número total de casos, em que o número de acertos do fenótipo com uso de marcadores moleculares corresponde à soma dos casos em que as cultivares ou as linhagens foram classificadas como resistentes pelos marcadores moleculares e foram resistentes na análise fenotípica ou em que foram classificadas como suscetíveis pelos marcadores moleculares e foram suscetíveis na análise fenotípica; e o número total de casos corresponde ao número total de genótipos considerados. 


\section{Resultados e Discussão}

A severidade da infecção por giberela variou de 1,95 a $41,3 \%$, na média dos dois anos, e foram observadas 30 linhagens e cultivares resistentes, 26 intermediárias e 40 suscetíveis (Figura 2). Entre os 19 marcadores analisados para os três possíveis QTLs, 12 foram polimórficos entre as linhagens e as cultivares estudadas (Tabela 2). O número de alelos observados variou de 2 a 4. Schuster et al. (2009), em estudo da diversidade genética de trigo no Brasil, encontraram 23 marcadores polimórficos, em um conjunto de 43 marcadores avaliados, além de variação de 2 a 5 alelos por marcador, em 36 cultivares de trigo. A partir dos resultados obtidos, é possível inferir que o germoplasma utilizado apresenta regiões conservadas no genoma e que não apresentam variabilidade. Isso reforça a necessidade de validação de marcadores moleculares, para avaliar sua utilidade na seleção assistida por marcadores moleculares, em programas de melhoramento.

$\mathrm{Na}$ avaliação da diferença de severidade entre os genótipos em cada marcador, os marcadores Xgwm533, no cromossomo 3B; Xbarc180, no cromossomo 5A; Wmc397, Xbarc24, Xbarc101 e Wmc398, no cromossomo 6B, apresentaram diferença significativa entre as médias dos alelos (Tabela 1). Os marcadores Xbarc180, no cromossomo 5A, e Wmc397, no cromossomo $6 \mathrm{~B}$, foram significativos a $1 \%$ de probabilidade na análise de correlação, tanto para a severidade quanto para a reação das variedades ou das linhagens à giberela (Figura 3). $\mathrm{O}$ marcador Xbarc101, no cromossomo $6 \mathrm{~B}$, foi associado apenas à severidade.

$\mathrm{Na}$ análise de regressão linear simples, os marcadores Xbarc180, no cromossomo 5A; Wmc397, Xbarc24, Xbarc101 e Wmc398, no cromossomo 6B, foram significativos a $5 \%$ de probabilidade. No entanto, os marcadores Xgwm389 e Xgwm533, ambos no cromossomo $3 \mathrm{~B}$, foram significativos apenas quanto à reação das plantas à giberela (Tabela 2).

Shi et al. (2008), ao avaliar uma população derivada da cultivar Sumai 3, encontraram associação da resistência do trigo à giberela com os marcadores Xgwm389, Xgwm533, Xbarc133, Xbarc147, Xbarc68 e Xgwm493, no cromossomo 3B; Xbarc101 e Wmc397, no cromossomo 6B; e Xgwm293, no cromossomo 5A. Desses, os marcadores Xgwm533, no cromossomo 3B; Xbarc101, Xgwm389 e Wmc397, no cromossomo 6B, foram significativamente associados à resistência à giberela no presente trabalho. Os marcadores Xbarc147 e Xgwm493, no cromossomo 3B, não foram associados à resistência; já os marcadores Xbarc133, no cromossomo 3B, e Xbarc293, no cromossomo 5A, foram monomórficos na população avaliada. Os autores identificaram o marcador Xbarc293 como associado à resistência no cromossomo $5 \mathrm{~A}$. No presente trabalho, o marcador Xbarc180, localizado próximo ao marcador Xbarc293 (Röder, 1998), foi associado à resistência no cromossomo 5A. Com os resultados obtidos, é possível inferir que os três QTLs, nos cromossomos 3B, 5A e $6 \mathrm{~B}$, estão presentes na população de variedades e linhagens em estudo.

Segundo Zhou et al. (2002) e Liu \& Anderson (2003), os marcadores no cromossomo 3B utilizados no presente trabalho estão ligados ao QTL Qfhs.ndsu-3BS. Este QTL corresponde ao gene Fhb1 e tem origem na cultivar Sumai 3, que é a principal fonte de resistência tipo II à giberela. Esse QTL no
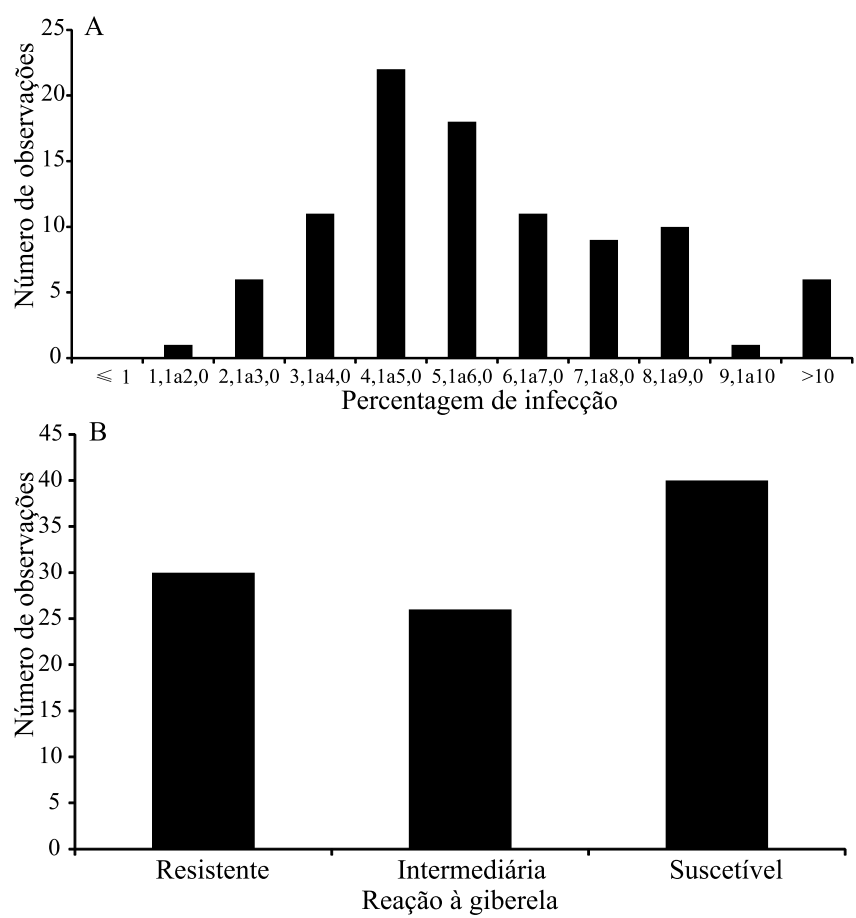

Figura 2. Distribuição de frequências das médias de dois anos das percentagens de severidade da giberela nas espigas de linhagens e variedades de trigo (Triticum aestivum), avaliadas em condições controladas de campo (A); e distribuição de frequência da classificação da reação das linhagens e das variedades de trigo à giberela (B). 
cromossomo 3B tem sido confirmado por diversos autores (Zhou et al., 2004; Ma et al., 2006; Pumphrey et al., 2007).

$\mathrm{O}$ cromossomo 6B apresenta o gene $F h b 2$ de resistência tipo II à giberela (Cuthbert et al., 2006). Somers et al. (2006) mapearam esse QTL na região do cromossomo 6B flanqueada pelos marcadores Xgwm518 e Xbarc125. Os marcadores Wmc398 e Wmc397, utilizados no presente trabalho, encontramse entre os marcadores Xgwm518 e Xbarc125 (Röder et al., 1998). Isso confirma que a região associada aos marcadores Wmc398 e Wmc397 identificada

Tabela 2. Análise de associação entre os marcadores moleculares microssatélites e a severidade da infecção por giberela nas plantas de trigo (Triticum aestivum) ${ }^{(1)}$.

\begin{tabular}{|c|c|c|c|c|c|c|c|c|c|c|c|}
\hline \multirow[t]{2}{*}{ Marcador } & \multirow[t]{2}{*}{ Cromossomo } & \multirow[t]{2}{*}{ Posição } & \multirow[t]{2}{*}{ Alelo } & \multirow{2}{*}{$\begin{array}{c}\text { Média da } \\
\text { severidade }\end{array}$} & \multirow{2}{*}{$\begin{array}{l}\text { Número de } \\
\text { observações }\end{array}$} & \multicolumn{3}{|c|}{ Regressão da reação } & \multicolumn{3}{|c|}{ Regressão da severidade } \\
\hline & & & & & & $\mathrm{R}^{2}(\%)$ & $\mathrm{P}(\%)$ & Inclinação $^{(2)}$ & $\mathrm{R}^{2}(\%)$ & $\mathrm{P}(\%)$ & Inclinação \\
\hline \multirow{4}{*}{ Xgwm389 } & \multirow{4}{*}{$3 \mathrm{~B}$} & \multirow{4}{*}{5,0} & 1 & $5,08 \mathrm{a}$ & 10 & 6,03 & 1,7 & $-0,33$ & 0,91 & 63,4 & $-0,86$ \\
\hline & & & 2 & $5,49 \mathrm{a}$ & 16 & 0,04 & 85,1 & $-0,02$ & 0,85 & 61,4 & $-0,68$ \\
\hline & & & 3 & $6,10 \mathrm{a}$ & 19 & 3,06 & 9,5 & 0,18 & 0,23 & 66,1 & $-0,33$ \\
\hline & & & 4 & $7,63 \mathrm{a}$ & 39 & 0,01 & 93,7 & 0,01 & 2,97 & 10,2 & 0,98 \\
\hline \multirow{3}{*}{ Xgwm533 } & \multirow{3}{*}{$3 \mathrm{~B}$} & \multirow{3}{*}{11,1} & 1 & 5,15 & 1 & - & - & - & - & - & - \\
\hline & & & 2 & $4,70 \mathrm{~b}$ & 18 & 3,22 & 13,3 & $-0,18$ & 4,49 & 7,5 & $-1,53$ \\
\hline & & & 3 & $7,40 \mathrm{a}$ & 44 & 6,27 & 4,0 & 0,24 & 4,29 & 9,1 & 1,14 \\
\hline \multirow{3}{*}{ Xbarc133 } & \multirow{3}{*}{$3 \mathrm{~B}$} & \multirow{3}{*}{15,6} & 1 & $5,57 \mathrm{a}$ & 24 & 0,38 & 57,9 & $-0,06$ & 2,62 & 13,0 & $-1,01$ \\
\hline & & & 2 & $5,55 \mathrm{a}$ & 19 & 0,31 & 61,3 & 0,05 & 3,72 & 6,8 & 1,23 \\
\hline & & & 3 & $6,95 \mathrm{a}$ & 17 & 0,10 & 76,4 & 0,03 & 0,02 & 88,5 & $-0,09$ \\
\hline \multirow{3}{*}{ Xbarc147 } & \multirow{3}{*}{$3 \mathrm{~B}$} & \multirow{3}{*}{16,1} & 1 & 7,72 & 2 & - & - & - & - & - & - \\
\hline & & & 2 & $6,37 \mathrm{a}$ & 27 & 0,03 & 86,7 & $-0,02$ & 0,01 & 92,7 & $-0,06$ \\
\hline & & & 3 & $6,76 \mathrm{a}$ & 58 & 0,36 & 57,0 & $-0,05$ & 0,03 & 85,7 & $-0,11$ \\
\hline \multirow{2}{*}{ Xgwm493 } & \multirow{2}{*}{$3 \mathrm{~B}$} & 216 & 1 & $5,66 \mathrm{a}$ & 38 & 3,15 & 9,2 & $-0,15$ & 6,66 & $1,4,0$ & $-1,50$ \\
\hline & & & 2 & $8,47 \mathrm{a}$ & 36 & 3,15 & 9,2 & 0,15 & 6,66 & $1,4,0$ & 1,50 \\
\hline & & & 1 & $5,17 \mathrm{a}$ & 6 & 1,42 & 25,4 & 0,20 & 0,50 & 50,7 & $-0,80$ \\
\hline Xbarc75 & $3 \mathrm{~B}$ & - & 2 & $7,48 \mathrm{a}$ & 54 & 0,33 & 59,5 & 0,05 & 3,16 & 9,0 & 1,04 \\
\hline & & & 3 & $5,48 \mathrm{a}$ & 28 & 1,54 & 24,2 & $-0,11$ & 2,15 & 16,4 & $-0,90$ \\
\hline & & & 1 & $5,12 b$ & 37 & 10,47 & 0,2 & $-0,28$ & 4,46 & 4,2 & $-1,21$ \\
\hline Xharc180 & $5 \mathrm{~A}$ & 111 & 2 & $6,09 \mathrm{ab}$ & 36 & 2,29 & 15,0 & 0,13 & 0,38 & 57,0 & $-0,35$ \\
\hline ADarciov & JA & 11,1 & 3 & $11,76 \mathrm{a}$ & 14 & 5,92 & 1,9 & 0,29 & 15,51 & 0,0 & 3,07 \\
\hline & & & 4 & 5,18 & 2 & - & - & - & - & - & - \\
\hline & & & 1 & $4,55 \mathrm{a}$ & 7 & 3,72 & 6,5 & $-0,30$ & 1,22 & 30,1 & $-1,17$ \\
\hline Youmm620 & 54 & 538 & 2 & $4,81 \mathrm{a}$ & 5 & 0,51 & 69,9 & $-0,08$ & 0,82 & 62,7 & $-0,20$ \\
\hline Agwmosy & JA & (25,0 & 3 & $5,10 \mathrm{a}$ & 9 & 0,15 & 83,3 & 0,04 & 1,38 & 54,4 & $-0,83$ \\
\hline & & & 4 & 6,01 & 2 & - & - & - & - & - & - \\
\hline & & & 1 & $10,43 a$ & 21 & 12,92 & 0,1 & 0,37 & 13,31 & 0,1 & 2,44 \\
\hline Wmc397 & $6 \mathrm{~B}$ & 37,3 & 2 & $6,68 b$ & 36 & 1,44 & 25,9 & $-0,11$ & 2,19 & 16,0 & $-0,85$ \\
\hline & & & 3 & $5,35 \mathrm{~b}$ & 31 & 3,74 & 6,4 & $--0,17$ & 2,83 & 10,9 & $-0,99$ \\
\hline & & & 1 & $7,14 \mathrm{a}$ & 8 & 0,15 & 71,0 & 0,05 & 0,19 & 67,3 & 0,40 \\
\hline Xharc24 & $6 \mathrm{~B}$ & 547 & 2 & $7,03 \mathrm{a}$ & 46 & 5,13 & 2,5 & 0,19 & 0,51 & 50,5 & 0,40 \\
\hline Xoarc 24 & OB & $34, I$ & 3 & $4,87 \mathrm{~b}$ & 28 & 4,58 & 3,4 & $-0,20$ & 4,11 & 4,5 & $-1,23$ \\
\hline & & & 4 & $8,50 \mathrm{a}$ & 12 & 0,70 & 57,7 & $-0,11$ & 1,68 & 20,5 & 1,08 \\
\hline & & & 1 & $13,02 \mathrm{a}$ & 6 & 5,39 & 2,6 & 0,39 & 9,01 & 0,4 & 3,42 \\
\hline Xharc101 & $6 \mathrm{~B}$ & 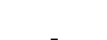 & 2 & 6,14 & 2 & - & - & - & - & - & - \\
\hline ADarciol & OD & - & 3 & $6,41 b$ & 53 & 0,85 & 56,5 & $-0,09$ & 2,80 & 14,5 & $-1,13$ \\
\hline & & & 4 & $7,50 \mathrm{~b}$ & 9 & 0,41 & 59,5 & $-0,08$ & 0,06 & 83,0 & 0,23 \\
\hline & & & 1 & $4,25 \mathrm{a}$ & 7 & 5,02 & 2,8 & $-0,37$ & 1,48 & 23,9 & $-1,29$ \\
\hline Wmc398 & $6 \mathrm{~B}$ & - & 2 & $5,53 \mathrm{ab}$ & 34 & 0,17 & 69,7 & $-0,04$ & 2,23 & 14,5 & $-0,86$ \\
\hline & & & 3 & $7,71 \mathrm{~b}$ & 50 & 2,48 & 12,3 & 0,14 & 4,35 & 4,0 & 1,17 \\
\hline
\end{tabular}

${ }^{(1)}$ Médias seguidas de letras iguais, dentro do mesmo marcador microssatélite, não diferem pelo teste de Tukey, a $5 \%$ de probabilidade. ${ }^{(2)}$ Coeficiente angular da equação de regressão linear. Sinal positivo na inclinação indica alelo associado à suscetibilidade, e sinal negativo indica alelo associado à resistência à giberela. 
na população em estudo está ligada ao gene $F h b 2$. Burlakoti et al. (2010) também encontraram associação dos marcadores Wmc397 e Wmc398 com esse QTL, em uma população derivada da cultivar Alsen.

O QTL no cromossomo 5A foi observado por Shen et al. (2003), Chrpová et al. (2011) e Xue et al. (2011) flanqueado pelos marcadores Xgwm304 e Xgwm415. Esse loco foi denominado de $F h b 5$ e está associado à resistência tipo I à giberela. O marcador Xbarc180, utilizado no presente trabalho, está localizado na região do marcador Xgwm304 (Song et al., 2005). Desse modo, pode-se inferir que, na população avaliada, o QTL no cromossomo 5A também está presente no loco Fhb5.

No presente trabalho, os marcadores Xbarc180, no cromossomo 5A; Wmc397 e Xbarc101, no cromossomo
6B, foram significativos em todas as análises. Contudo, os marcadores Xgwm533, no cromossomo 3B; Xbarc180, no cromossomo 5A; Wmc397, Xbarc24 e Wmc398, no cromossomo 6B, foram significativos pelo teste de Tukey e pela análise de regressão linear. Isso pode ser explicado pelo nível de significância de $1 \%$ utilizado na análise de correlação. Ao nível de significância de $5 \%$, os mesmos marcadores são significativos em todas as análises $[\operatorname{com}-\log (\mathrm{P})=$ 1,3] (Figura 3). Esses marcadores podem ser úteis na seleção de plantas contendo QTLs de resistência do trigo à giberela, em programas de melhoramento.

Alguns marcadores apresentam alelos especificamente associados à resistência, enquanto outros marcadores apresentam alelos associados especificamente à suscetibilidade do trigo à giberela.

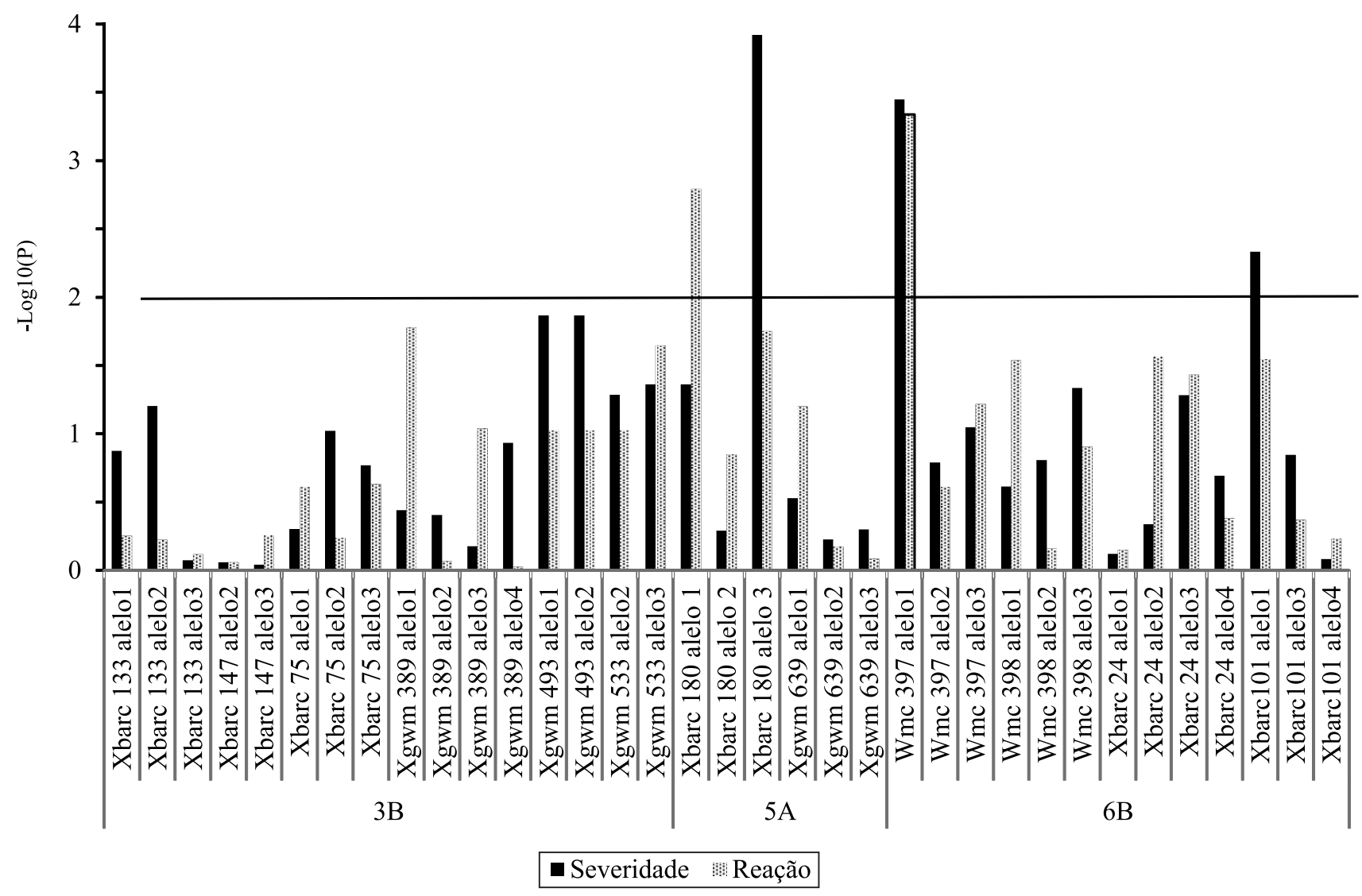

Figura 3. Probabilidades associadas à análise de correlação dos alelos de cada marcador com a severidade e com a reação das linhagens e das variedades de trigo (Triticum aestivum) à giberela. As probabilidades estão apresentadas na escala de $-\log 10(\mathrm{P})$. A linha horizontal indica o ponto de corte para $1 \%$ de probabilidade. 3B, 5A e $6 \mathrm{~B}$ identificam os cromossomos onde os marcadores estão localizados. 
Nos casos de alelos associados à suscetibilidade, a presença desses alelos resulta em uma severidade maior da giberela; já os demais alelos, no mesmo loco, apresentam menor severidade, mesmo com alelos diferentes. Nos alelos 1 e 3, respectivamente, dos marcadores Xbarc180, no cromossomo 5A, e Xbarc24, no cromossomo 6B, observou-se coeficiente angular negativo para a severidade e para a reação à giberela, o que é indicativo de que esses alelos estão associados à resistência das plantas à doença. Os alelos 1 dos marcadores Xbarc389, no cromossomo 3B, e Wmc398, no cromossomo $6 \mathrm{~B}$, apresentaram coeficiente angular negativo para a reação, o que também é indicativo de que esses alelos estão associados à resistência. Entretanto, os alelos 3, 1 e 1, respectivamente, dos marcadores Xbarc180, no cromossomo 5A; Wmc397 e Xbarc101, no cromossomo $6 \mathrm{~B}$, apresentaram coeficiente angular positivo tanto para a severidade quanto para a reação, o que é indicativo de que esses alelos estão associados à suscetibilidade do trigo à giberela. Os alelos $3 \mathrm{e}$ 2, respectivamente, dos marcadores Xgwm533, no cromossomo 3B, e Xbarc24, no cromossomo 6B, apresentaram coeficiente angular positivo apenas para severidade, enquanto o alelo 3 do marcador Xbarc398, no cromossomo $6 \mathrm{~B}$, apresentou coeficiente angular positivo para a severidade. Esses alelos também estão associados à suscetibilidade do trigo à giberela (Tabela 2). Nesses casos, a seleção deve ser realizada contra esses alelos.

Ao se considerar a reação das plantas à giberela, os marcadores significativos no cromossomo 3B explicaram 6,03\% (Xbarc389) e 6,27\% (Xgwm533) da variação observada; já para severidade, nenhum marcador no cromossomo $3 \mathrm{~B}$ foi significativo (Tabela 2). Anderson et al. (2001), Zhou et al. (2002) e Shen et al. (2003) relataram variação de 24,8 a $41,6 \%, 18$ a $48 \%$ e 19,2 a $40 \%$ no efeito do QTL no cromossomo $3 \mathrm{~B}$, respectivamente.

No cromossomo 5A, o marcador Xbarc180 explicou $10,47 \%$ da variação na reação e $15,51 \%$ na variação da severidade da doença. Gervais et al. (2003) observaram variação de 10,1 a $18,6 \%$ no efeito do QTL no cromossomo 5A. No cromossomo 6B, os marcadores Wmc398, Xbarc24, Xbarc101 e Wmc397 explicaram 5,02, 5,13, 5,39 e 12,92\%, respectivamente, da variação na reação, e 4,35, 4,11, 9,01 e $13,31 \%$ da variação na severidade, respectivamente. Anderson et al. (2001), Shen et al. (2003) e Somers et al. (2006) constataram efeitos de 4,9 a $9,2 \%$, 2,9 a $3,4 \%$ e $23 \%$, respectivamente, para o QTL no cromossomo $6 \mathrm{~B}$.

Os marcadores significativos na análise de correlação foram utilizados para a análise de regressão múltipla. Para a severidade da doença, os alelos 3 e 1, respectivamente, dos marcadores Xbarc180 e Wmc397 explicaram $29,29 \%$ da variação observada, sendo que esses alelos estão associados à suscetibilidade das plantas à giberela. Para a reação das plantas à giberela, os alelos 3 e 1, respectivamente, dos marcadores Wmc397 e Xbarc101 explicaram 14,25\% da variação, sendo que o alelo 3 do marcador Wmc397 está associado à resistência, e o alelo 1 do marcador Xbarc101 está associado à suscetibilidade.

Ao se selecionar apenas os genótipos com a presença do alelo 3 do marcador Wmc397 e com a ausência do alelo 1 do marcador Xbarc101, 15 genótipos foram considerados resistentes; 8 genótipos, suscetíveis; e 5, intermediários. A média de severidade dos genótipos selecionados por essa combinação de marcadores e alelos foi de 5,1. Ao contrário, ao se selecionar para a ausência do alelo 3 do marcador Wmc397 e a presença do alelo 1 do marcador Xbarc101, dois genótipos foram suscetíveis, e um intermediário. Nesta combinação, nenhum genótipo foi resistente. A média de severidade com essa combinação de alelos foi de 18,5. Ao se considerar apenas os genótipos resistentes e suscetíveis, a eficiência de seleção foi de $68 \%$ para a reação do trigo à giberela.

$\mathrm{Na}$ população avaliada, o QTL presente no cromossomo $6 \mathrm{~B}$, possivelmente $F h b 2$, é responsável pela maior parte da resistência das plantas à giberela, ao contrário do que é comumente relatado na literatura. Anderson et al. (2001), Shen et al. (2003) e Burlakoti et al. (2010) verificaram efeitos elevados para o loco $F h b 1$, no cromossomo 3B, e efeitos menores para o loco Fhb2, no cromossomo 6B. O menor efeito do QTL no cromossomo 3B, no presente trabalho, pode ser atribuído à maior distância entre os marcadores utilizados no cromossomo 3B e o QTL ou à baixa frequência desse QTL na população utilizada.

No presente trabalho, foram avaliadas três regiões cromossômicas do trigo, nos cromossomos 3B, $5 \mathrm{~A}$ e $6 \mathrm{~B}$, para resistência à giberela em condições controladas de campo. As três regiões estão associadas à resistência (Tabela 2), mas os QTLs nos cromossomos $6 \mathrm{~B}$ e $5 \mathrm{~A}$ têm maior efeito. A avaliação de um conjunto 
de germoplasma em vez de uma população estruturada possibilitou a avaliação de vários alelos por loco, o que permitiu identificar os alelos associados à resistência e os alelos associados à suscetibilidade. A utilização desse tipo de população não estruturada permite validar os marcadores moleculares com uma avaliação mais completa dos alelos em cada loco. Sabe-se que a utilização de populações segregantes derivadas de cruzamentos controlados permite avaliar apenas dois alelos em cada loco, enquanto um conjunto de germoplasma permite avaliar o efeito de todos os alelos presentes na população utilizada.

A utilização da seleção com esses marcadores moleculares aumenta significativamente a frequência de plantas resistentes no conjunto de plantas selecionadas. Para eliminar completamente as linhagens suscetíveis, pode-se utilizar o método sequencial, ao se selecionar inicialmente as linhagens com os marcadores moleculares, o que elimina grande parte das plantas suscetíveis. Em seguida, as plantas selecionadas pelos marcadores moleculares podem ser submetidas à avaliação fenotípica, para eliminar as plantas suscetíveis que foram selecionadas pelos marcadores. Com isso, diminui-se bastante a quantidade de plantas a serem avaliadas fenotipicamente.

\section{Conclusões}

1. Os marcadores moleculares localizados nas regiões dos cromossomos $3 \mathrm{~B}, 5 \mathrm{~A}$ e $6 \mathrm{~B}$ são válidos para seleção quanto à resistência do trigo (Triticum aestivum) à giberela, no germoplasma avaliado.

2. Os marcadores Wmc397, Xbarc180 e Xbarc101 podem ser utilizados para a seleção assistida por marcadores moleculares, para resistência à giberela.

\section{Agradecimentos}

À Coordenação de Aperfeiçoamento de Pessoal de Nível Superior (Capes), pela concessão de bolsa e à Cooperativa Central de Pesquisa Agrícola (Coodetec), pelo apoio financeiro.

\section{Referências}

ANDERSON, J.A.; CHAO, S.; LIU, S. Molecular breeding using a major QTL for Fusarium head blight resistance in wheat. Crop Science, v.47, p.S112-S119, 2007. DOI: 10.2135/ cropsci2007.04.0006IPBS.
ANDERSON, J.A.; STACK, R.W.; LIU, S.; WALDRON, B.L.; FJELD, A.D.; COYNE C.; MORENO-seVILLA, B.; FETCH, J.M.; SONG, Q.J.; CREGAN, P.B.; FROHBERG, R.C. DNA markers for Fusarium head blight resistance QTLs in two wheat populations. Theoretical and Applied Genetics, v.102, p.1164-1168, 2001. DOI: $10.1007 / \mathrm{s} 001220000509$.

BUERSTMAYR, H.; LEMMENS, M.; HARTL, L.; DOLDI, L.; STEINER, B.; STIERSCHNEIDER, M.; RUCKENBAUER, P. Molecular mapping of QTLs for Fusarium head blight resistance in spring wheat. I. Resistance to fungal spread (type II resistance). Theoretical and Applied Genetics, v.104, p.84-91, 2002. DOI: $10.1007 / \mathrm{s} 001220200009$.

BURLAKOTI, R.R.; MERGOUM, M.; SHAHRYAR, F.K.; ADHIKARI, T.B. Combining different resistance components enhances resistance to Fusarium head blight in spring wheat. Euphytica, v.172, p.197-205, 2010. DOI: 10.1007/ s10681-009-0035-0.

CASA, R.T.; REIS, E.M.; BLUM, M.M.C.; BOGO, A.; SCHEER, O.; ZANATA, T. Danos causados pela infecção de Giberella zeae em trigo. Fitopatologia Brasileira, v.29, p.289-293, 2004. DOI: 10.1590/S0100-41582004000300008.

CHRPOVÁ, J.; SIP, V.; SEDLACEK, T.; STOCKOVA, L.; VESKRNA, O.; HORCICKA, P. Effectiveness of marker-based selection for Fusarium head blight resistance in spring wheat. Czech Journal of Genetics and Plant Breeding, v.47, p.S123-S129, 2011.

CRUZ, C.D. Programa Genes: biometria. Viçosa: Ed. da UFV, 2006. 382p.

CUTHBERT, P.A.; SOMERS, D.J.; THOMAS, J.; CLOUTIER, S.; BRULÉ-BABEL, A. Fine mapping $F h b 1$, a major gene controlling fusarium head blight resistance in bread wheat (Triticum aestivum L.). Theoretical and Applied Genetics, v.112, p.1465-1472, 2006. DOI: 10.1007/s00122-006-0249-7.

DRAEGER, R.; GOSMAN, N.; STEED, A.; CHANDLER, E.; THOMSETT, M.; SRINIVASACHARY; SCHONDELMAIER, J.; BUERSTMAYR, E.; LEMMENS, M.; SCHMOLKE, M.; MESTERHAZY, A.; NICHOLSON, P. Identification of QTLs for resistance to Fusarium head blight, DON accumulation and associated traits in the winter wheat variety Arina. Theoretical and Applied Genetics, v.115, p.617-625, 2007. DOI: 10.1007/ s00122-007-0592-3.

GERVAIS, L.; DEDRYVER, F.; MORLAIS, J.-Y.; BODUSSEAU, V.; NEGRE, S.; BILOUS, M.; GROOS, C.; TROTTET, M. Mapping of quantitative trait loci field resistance to Fusarium head blight in a European winter wheat. Theoretical and Applied Genetics, v.106, p.961-970, 2003.

GOSMAN, N.; BAYLES, R.; JENNINGS, P.; KIRBY, J.; NICHOLSON, P. Evaluation and characterization of resistance to fusarium head blight caused by Fusarium culmorum in UK winter wheat cultivars. Plant Pathology, v.56, p.264-276, 2007. DOI: 10.1111/j.1365-3059.2006.01508.x.

LARGE, E.C. Growth stages in cereals: illustration of the Feekes scale. Plant Pathology, v.3, p.128-129, 1954. DOI: 10.1111/ j.1365-3059.1954.tb00716.x.

LIN, F.; KONG, Z.X.; ZHU, H.L.; XUE, S.L.; WU, J.Z.; TIAN, D.G.; WEI, J.B.; ZHANG, C.Q.; MA, Z.Q. Mapping QTL 
associated with resistance to Fusarium head blight in the Nanda2419 $\mathrm{x}$ Wangshuibai population. I. Type II resistance. Theoretical and Applied Genetics, v.109, p.1504-1511, 2004. DOI: 10.1007/ s00122-004-1772-z.

LIU, S.; ANDERSON, J.A. Marker assisted evaluation of Fusarium head blight resistant wheat germplasm. Crop Science, v.43, p.760-766, 2003. DOI: 10.2135/cropsci2003.0760.

LIU, S.; HALL, M.D.; GRIFFEY, C.A.; MCKENDRY, A.L. Meta-analysis of QTL associated with Fusarium head blight resistance in wheat. Crop Science, v.49, p.1955-1968, 2009. DOI: 10.2135/cropsci2009.03.0115.

MA, H.X.; ZHANG, K.M.; GAO, L.; BAI, G.H.; CHEN, H.G.; CAI, Z.X.; LU, W.Z. Quantitative trait loci for resistance to fusarium head blight and deoxynivalenol accumulation in Wangshuibai wheat under field conditions. Plant Pathology, v.55, p.739-745, 2006. DOI: 10.1111/j.1365-3059.2006.01447.x.

PUMPHREY, M.O.; BERNARDO, R.; ANDERSON, J.A. Validating the Fhb1 QTL for Fusarium head blight resistance in near-isogenic wheat lines developed from breeding populations. Crop Science, v.47, p.200-206, 2007. DOI: 10.2135/ cropsci2006.03.0206.

RÖDER, M.S.; KORZUM, V.; WENDEHAKE, K.; PLASCHKE, J.; TIXIER, M.-H.; LEROY, P.; GANAL, M.W. A microsatellite map of wheat. Genetics, v.149, p.2007-2023, 1998.

SCHUSTER, I. Seleção assistida por marcadores moleculares para QTLs no melhoramento genético. In: CARDOSO, D.L.; LUZ, L.N. da; PEREIRA, T.N.S. (Ed.). Estratégias em melhoramento de Plantas. Viçosa: Arka, 2011. p.72-81.

SCHUSTER, I.; VIEIRA, E.S.N.; SILVA, G.J. da; FRANCO, F.A.; MARCHIORO, V.S. Genetic variability in Brazilian wheat cultivars assessed by microsatellite markers. Genetics and Molecular Biology, v.32, p.557-563, 2009. DOI: 10.1590/ S1415-47572009005000045.

SHEN, X.; ITTU, M.; OHM, H.W. Quantitative trait loci conditioning resistance to Fusarium head blight in wheat line F201R. Crop Science, v.43, p.850-857, 2003. DOI: 10.2135/ cropsci2003.8500.

SHI, J.R.; XU, D.H.; YANG, H.Y.; LU, Q.X.; BAN, T. DNA marker analysis for pyramided of Fusarium head blight (FHB) resistance QTLs from different germplasm. Genetica, v.133, p.77-84, 2008. DOI: $10.1007 / \mathrm{s} 10709-007-9186-x$.
SOMERS, D.J.; FEDAK, G.; CLARKE, J.; CAO, W.G. Mapping of FHB resistance QTLs in tetraploid wheat. Genome, v.49, p.1586-1593, 2006. DOI: 10.1139/g06-127.

SONG, Q.J.; SHI, J.R.; SINGH, S.; FICKUS, E.W.; COSTA, J.M.; LEWIS, J.; GILL, B.S.; WARD, R.; CREGAN, P.B. Development and mapping of microsatellite (SSR) markers in wheat. Theoretical and Applied Genetics, v.110, p.550-560, 2005. DOI 10.1007/ s00122-004-1871-x.

STACK, R.W.; MCMULLEN, M.P. A visual scale to estimate severity of Fusarium head blight in wheat. Fargo: North Dakota State University Extension Service, 1995. 2p. (NSDU. Extension Publication PP-1095).

UNITED STATES DEPARTAMENT OF AGRICULTURE. GrainGenes: a database for triticale and Avena. Available at: $<$ http://wheat.pw.usda.gov/GG2/index.shtml $>$. Accessed on: 16 Nov. 2014.

XUE, S.; LI, G.; JIA, H.; LIN, F.; CAO, Y.; XU, F.; TANG, M.; WANG, Y.; WU, X.; ZHANG, Z.; ZHANG, L.; KONG, Z.; MA, Z. Marker-assisted development and evaluation or near-isogenic lines for scab resistance QTLs of wheat. Molecular Breeding, v.25, p.397-405, 2010. DOI: 10.1007/s11032-009-9339-y.

XUE, S.; XU, F.; TANG, M.; ZHOU, Y.; LI, G.; AN, X.; LIN, F.; XU, H.; JIA, H.; ZHANG, L.; KONG, Z.; MA, Z. Precise mapping Fhb5, a major QTL conditioning resistance to Fusarium infection in bread wheat (Triticum aestivum L.). Theoretical and Applied Genetics, v.123, p.1055-1063, 2011. DOI: 10.1007/ s00122-011-1647-z.

ZHOU, W.-C.; KOLB, F.L.; BAI, G.-H.; DOMIER, L.L.; BOZE, L.K.; SMITH, N.J. Validation of a major QTL for scab resistance with SSR markers and use of marker-assisted selection in wheat. Plant Breeding, v.122, p.40-46, 2003. DOI: 10.1046/j.14390523.2003.00802.x.

ZHOU, W.C.; KOLB, F.L.; BAI, G.H.; SHANER, G.; DOMIER, L.L. Genetic analysis of scab resistance QTL in wheat with microsatellite and AFLP markers. Genome, v.45, p.719-727, 2002. DOI: $10.1139 / \mathrm{g} 02-034$.

ZHOU, W.C.; KOLB, F.L.; YU, J.B.; BAI, G.; BOZE, L.K.; DOMIER, L.L. Molecular characterization of Fusarium head blight resistance in Wangshuibai with simple sequence repeat and amplified fragment length polymorphism markers. Genome, v.47, p.1137-1143, 2004. DOI: 10.1139/g04-069.

Recebido em 2 de julho de 2014 e aprovado em 4 de dezembro de 2014

Pesq. agropec. bras., Brasília, v.50, n.1, p.63-72, jan. 2015

DOI: $10.1590 / \mathrm{S} 0100-204 X 2015000100007$ 